\title{
The Fourth Meeting of the Committee of European Solar Radio Astronomers
}

\author{
Berne, Switzerland, 5-7 March 1974
}

This was the first meeting of the Committee of European Solar Radio Astronomers (CESRA) that has been sponsored by EPS. Another noteworthy circumstance is the fact that this meeting was combined with one of the regular meetings of the Joint Organization for Solar Observations (JOSO). As a consequence, solar astronomers of different specialisms were brought together and both scientific and organizational matters were discussed in two common sessions. Host to the meeting was the Institut für angewandte Physik through the courtesy of its director K. P. Meyer; E. Schanda was the most efficient coordinator of the local organizing team. Some 80 participants attended the combined meeting.

CESRA is a regional organization of solar radio astronomers with the purpose to stimulate cooperation and to indicate and help realize possibilities for joint efforts and common research projects.

JOSO aims at providing optical solar astronomers of adhering countries with a common observatory at a site specially selected for making high resolution observations of the solar surface. An elaborate site testing programme is being jointly conducted already for some 4 years and it is expected that the final choice of a site will be made within two years from now.

The CESRA meeting began with two invited lectures. Z. Svestka (Fraunhofer Institute, Freiburg im Breisgau) reviewed the results obtained recently with X-ray photography of the Sun, notably during the skylab mission. Particularly striking is the discovery of bright points, scattered all over the solar disk. These points seem to cor- respond with newly formed small bipolar features that can be identified optically.

The other invited lecture was given by M. R. Kundu (University of Maryland) who is endeavouring to apply interferometer systems, that are commonly in use for galactic radioastrono$\mathrm{my}$, to map the Sun at $\mathrm{cm}$ and $\mathrm{mm}$ wavelengths. This program yields information on the existence of finestructure in radio plages associated with centres of activity and in sources of flare-associated microwave bursts. These exploratory studies are very valuable for rating the scientific importance of a large microwave heliograph as it is being conceived by a working group for a Joint Interferometer Project (JIP). The chairman of this working group, E. Schanda from Berne, reported on the preliminary work devoted to this project. Scientific motivations have been collected in a booklet and are open for discussion by the solar community. High resolution microwave heliography will permit to study the evolution of magnetic structures in solar active regions in a novel way. It will thus provide a new tool for the investigation of the circumstances under which solar flares originate and, as a result, will shed light on their as yet poorly understood mechanism.

The JIP-project now calls for detailed studies like numerical work on possible antenna configurations and experimental tests (with the use of lasers) of such configurations. Valuable experience is being obtained from work at Bordeaux with a 2-element interferometer operating at 8.6 $\mathrm{mm}$.

In the same sphere of interest was the invited lecture of $\mathrm{M}$. Blum from

SENIOR SCIENTIST to contribute to Europe's first manned space flight programme (SPACELAB).

At the EUROPEAN SPACE RESEARCH AND TECHNOLOGY CENTRE (ESTEC), Noordwijk, the Netherlands, a post exists for a Ph. D. level physicists or astro-physicist with at least 7 years experience of space research hardware to act as co-ordinator of designated Spacelab payloads in Europe and to liaise with corresponding experimenters in the U.S.A.

The main tasks will include ensuring both adaptability of experiments to the engineering constraints of Spacelab and adaptation of Spacelab design to the best advantage of the experiments.

For particulars apply to: Head of Personnel, ESTEC, Domeinweg, Noordwijk, the Netherlands.
Meudon, who gave a review on millimeter observing techniques. In particular the limitations set by the atmosphere and the present state of the art of building large apertures suitable for $\mathrm{mm}$-wavelengths were discussed. Site testing is under way for the location of a great millimetric interferometer that is planned to comprise 4 antennas of 10 meter diameter for operation between 22 and $150 \mathrm{GHz}$. The instrument is meant mainly for galactic microwave spectroscopy.

In the combined CESRA/JOSO scientific session two invited papers were given by L. D. de Feiter and $M$. Kuperus, both from the Astronomical Institute at Utrecht. The first speaker dealt with integrated solar flare observations. During the flare process radiation and particles are produced that cover a large range of energies. Typical energies of electrons and of protons are $40 \mathrm{keV}$ and $1 \mathrm{MeV}$ respectively. Although particles may escape from the sun and cause terrestrial effects like, for instance, polar cap absorption, most of them remain trapped in coronal magnetic fields, thereby producing radio continuum emission and, on entering the chromosphere, $\mathrm{X}$ - and UV-emission.

Kuperus discussed the evolution of magnetic fields and unstable solar plasmas. He pointed out the possibilities of reconnection of magnetic lines of force and of annihilation of fields. Of particular interest are the processes that may occur along a neutral sheet. The tearing mode instability may lead to a change of magnetic topology. In connection with the energy release on the occasion of a flare, the kink instability offers interesting possibilities.

The contributed papers were arranged in three groups: observational, technical and theoretical. Observational papers dealt with a variety of subjects, including studies of decametric storms, microwave studies of solar active regions and polarization studies. Technical papers were given on microwave interferometry, digitization of a radiospectrograph and on questions of minimum redundancy in interferometry. Radiation and excitation mechanisms of type IV (continuum) and type III (fast drift) radiobursts were treated, among other topics, in the theoretical section. 
The present state of solar radio astronomy may be characterized as follows. The radio phenomena provide evidence of processes in a magnetoplasma that pose fundamental problems to plasma physics. Already theory seems to get some grip on what is going on. On the other hand, cleverly chosen and sophisticated observing techniques may provide us with meaningful data that yield clues to the explanation of facts that are still poorly understood. In particular, microwave radio bursts originate at the very levels where solar particles are accelerated. Better knowledge about their sources, to be gained by detailed heliography, may shed light on the nature of the flare process. Once this process is properly understood, valuable inferences might be drawn on the way of production of energetic particles in cosmic objects, like for instance quasars.

Proceedings of the CESRA meeting will be issued by the Institut für angewandte Physik at Bern under the editorship of $\mathrm{E}$. Schanda.

The JOSO program was mainly devoted to working sessions on practical matters like site testing campaigns, planning of activities, instrumental devices etc. There were two more general lectures. E. H. Schröter (Göttingen) comprehensively reviewed "The trends and problems of optical solar research", discussing the topics: the inhomogeneous photosphere, the convection zone, the heating of the corona, the differential rotation and the dynamo mechanism. C. Zwaan (Utrecht) dealt with the question "Why optical solar astronomy?", discussing some general astrophysical aspects of the photosphere and the chromosphere. These layers respond to the convection zone underneath and connect the corona with the convection zone by the transmission of energy fluxes and by magnetic structures.

The activities of JOSO are currently reported in JOSO's Annual Reports, issued by the Astronomical Institute at Utrecht under the editorship of $\mathrm{C}$. de Jager.

In the common organizational session it was agreed to create a commission that shall have the specific task to prepare a general solar meeting early in 1975. At this meeting a more permanent committee shall have to be formed which should promote the sake of solar research in Europe and reinforce the coherence of the efforts that are put into solar research projects.

A. D. Fokker jr., Utrecht

\section{Society News}

\section{Individual Ordinary Members}

Individual Ordinary Members in Belgium have the possibility to pay their membership fee to EPS through the Treasurer of the Belgian Physical Society, C. Henriet-Iserentant, I.N.W., Natuurkundig Laboratorium, Proeftuinstraat 86 , B-9000 Ghent, bFr. 320.corresponding to SFr. 24.-

\section{Member Societies}

I. Solomon, Paris has been elected as President of the French Physical Society to replace R. Castaign.

In the Polish Physical Society $Z$. Wilhelmi, Warsaw has been elected as President to succeed W. Rubinowicz, who was elected as Honorary President. The new General Secretary to take over from $\mathrm{H}$. Checinska is P. Decowski, Warsaw.

\section{Associate Members}

The following organizations have decided to join the European Physical Society as Associate Members:

Central Bureau for Nuclear Measurements, Geel, Belgium

Kernforschungsanlage Jülich $\mathrm{GmbH}$, Jülich, Federal Republic of Germany.

\section{Divisions}

Council accepted the rules of the former Physics in Astronomy Division and formally accepted its new name as Astronomy and Astrophysics Division.

The chairman of the Magnetism Section of the Condensed Matter Division informs all those intending to participate in the Europhysics Conference on the Physics in High Magnetic Fields, the section organizes in Grenoble, France, of the new dates for the conference. The meeting will start on Wednesday, 18 September 1974 at 3 p.m. and end on Saturday, 21 September at noon.

Fausto Fumi, Genoa, was elected as acting Chairman of the Board of the Metals Section of the Condensed Matter Division to replace A.R. Mackintosh.

The new Board of the Nuclear Physics Division elected C. van der Leun, Utrecht as its new Chairman.

The European Physical Society informs its members with regret of the death of Leon Rosenfeld, Copenhagen. He participated in the preparatory meetings leading to the formation of the Society.

\section{A CHOICE FROM OUR 1974 PHYSICS PUBLISHING PROGRAM}

\section{HAMILTON}

The Electromagnetic Interaction in Nuclear Physics

\section{ALDER/WINTHER}

Electromagnetic Excitation

JOACHAIN

Quantum Collision Theory

HORTON/MARADUDIN

Dynamical Properties of

Solids (I, II, and III)

SCHUTTE

The Theory of Molecular

Spectroscopy, Volume I

WOLF

Progress in Optics, Volume XII

BLINC/ZEKS

Lattice Dynamics of Ferroelectrics and Antiferroelectrics $\mathrm{BLOCH}$

Selected Works of Claude Bloch

BRUSSAARD/CLAUDEMANS Nuclear Shell Model

Applications

SHENOY/WAGNER

Mössbauer Isomer Shifts

McGOWAN/JOHN

The Applications of Gaseous Electronics

PRINS

Proceedings of the Van der Waals Centennial Conference on

Statistical Mechanics,

Amsterdam, August 27-31, 1973

(Special issue of the journal Physica)

For further details please write to:

North-Holland Publishing Co. P.O. Box 211 Amsterdam The Netherlands

Sole distributors for the USA and Canada.

\section{American Elsevier} Publishing Company 52 Vanderbilt Avenue New York, N.Y. 10017 